\title{
Real-time intelligent image processing for the internet of things
}

\author{
Mu-Yen Chen ${ }^{1} \cdot \mathrm{Hsin}-\mathrm{Te} \mathrm{Wu}^{2}$
}

Published online: 19 July 2021

(c) The Author(s), under exclusive licence to Springer-Verlag GmbH Germany, part of Springer Nature 2021

In recent years, Artificial Intelligence (AI) has been integrated into a large and rapidly expanding range of applications. Increased hardware processing speeds, combined with deep learning and machine learning technologies have allowed the development of real-time intelligent image processing at network edge regions. AI applications for intelligent image processing include handwriting recognition, image captioning and autonomous vehicle navigation. In addition, AI has been increasingly integrated into Internet of Things (IoT) applications. Some of these so-called AIoT applications include intelligent image processing in smart factories to monitor machinery conditions and control raw material inventory, identifying abnormalities in medical images, and automatic real-time scanning and recognition of license plates in traffic to locate stolen cars. These intelligent image processing applications of AIoT can improve quality of life and raise business competitiveness. This special issue focuses on such applications for the real-time use of AIoT intelligent image processing.

The topics covered in this special issue include (i) intelligent image processing applications and services to fulfill the real-time processing and performance demands, (ii) real-time deep learning and machine learning solutions to improve computational speed and increase recognition rates at network edges, (iii) new frameworks to optimize real-time AIoT image processing, and (iv) combining intelligent realtime image processing with edge computing, fog computing, and relevant techniques to balance the computational workloads between IoT devices and the server side.

$\mathrm{Hsin}-\mathrm{Te} \mathrm{Wu}$

hsinte@niu.edu.tw

Mu-Yen Chen

mychen119@gs.ncku.edu.tw

1 Department of Engineering Science, National Cheng Kung University, Tainan, Taiwan

2 Department of Computer Science and Information Engineering, National Ilan University, Yilan, Taiwan
The first theme of this special issue focuses on "Theories, models, and algorithms". Fan and Guan [1] have developed a deep face verification framework based on SIFT (scale invariant feature transform) and $\mathrm{CNN}$ (convolutional neural network) methods. Their experimental results show how the proposed model outperformed some state-of-theart methods on the LFW (Labeled Faces in the Wild) and YTB (YouTube) datasets. Su et al. [2] present a novel CNN framework based on a histogram of oriented gradients and a support vector machine (HOG-SVM) for real-time video recognition. Their experimental results indicate the proposed personal-value-based competency model can be used as an effective screening method replacing traditional approaches. Chang et al. [3] present a novel approach named Metric Learning to construct a Visual Simultaneous Localization and Mapping (VSLAM) method for localization and mapping. The proposed metric learning-based closed loop detection approach can be effectively applied to the TUM dataset. Neff et al. [4] have developed the EfficientHRNet consisting of three sub-networks including a backbone network, a highresolution network, and a heatmap prediction network. The proposed architecture can detect and perform multi-person human pose estimators in real-time on resource-constrained devices. Their performance evaluation show the proposed model outperformed ResNet for the ImageNet, CIFAR-100, and COCO2017 datasets. Singh et al. [5] have adopted both a wavelet-based solution and a multi-step image fusion technique to improve the image fusion process' efficiency. Their experimental results indicate that the wavelet and noise diffusion-based approaches are successful for image fusion.

The second theme of this issue focuses on "Optimization algorithms for AIoT". Gan et al. [6] have presented a hybrid intelligent collision detection algorithm based on a quantum behavior particle swarm optimization (PSO) algorithm and a differential algorithm. The proposed algorithm enhances collision detection efficiency and also improves the accuracy rate for behavior control in $3 \mathrm{D}$ animation interactive systems. Bacanin et al. [7] have designed a modified firefly algorithm (mFA) to optimize the CNN model hyperparameter settings. Using the IXI and TCIA datasets for MR 
images, their simulation results are promissing compared to other state-of-art methodologies.

The third theme of this issue covers "Intelligent real-time image processing and its extended fields". Chagas et al. [8] have designed twelve different $\mathrm{CNN}$ architectures, which are integrated with k-Nearest Neighbor (kNN), Naive Bayes, Random Forest, Multilayer Perceptron (MLP), and Support Vector Machine (SVM) for ImageNet dataset recognition. Their experimental results indicate the VGG19 model with the SVM classifier resulting as the best approach for pneumonia detection. Tong and Sun [9] have developed a realtime image detection and processing platform (RT-IDPP) based on back propagation neural network (BPNN) and SVM, while finding the proposed RT-IDPP achieves high image detection accuracy and tracking in real-time IoT environments. Ahmad et al. [10] have designed a real-time IoT-enabled people detection system based on the Cascade RCNN model by using the transfer learning architecture to improve the achieved 0.96 accuracy for the COCO dataset. Jhong et al. [11] have presented a nighttime object detection scheme based on a lightweight Deep Learning (DL) model on the Jetson TX2 edge device. A total of 19,030 images were collected, and 83,530 vehicles and 58,172 pedestrians were labeled. Their experimental results indicate the proposed system can provide high-accuracy for real-time object detection for applications of the Internet of Vehicles (IoV).

Overall, the eleven papers appearing in this special issue demonstrate multiple perspectives and approaches with implications for the theories, models, and algorithms used in real-time image processing and its IoT applications. These papers identify frameworks and techniques for artificial intelligence and deep learning, helping the field to advance towards a better understanding of big stream data pre-processing and real-time image analysis. It is seen that deep learning models provide a breakthrough in utilizing transfer learning theories and can successfully applied in real-time image analysis.

Future research on deep learning models needs to consider important issues such as optimizing neural network architectures and the hyperparameter settings that link them together. Furthermore, given the increasing popularity of smart city applications, an improved understanding of such processes could enhance the ICT (information and communication technology) impact on living environments for IoT and IoV applications. In summary, this special issue presents new real-time solutions for intelligent image processing within the context of IoT systems.

We would like to thank all the contributors of this special issue for their excellent collaboration and valuable scientific contributions. The quality of their research and their passion for conducting socially meaningful science is reflected in every paper of this issue. We are confident that readers of JRTIP and researchers working in the real-time image processing area find this special issue of great benefit.

\section{References}

1. Fan, Z., Yp, G.: A deep learning framework for face verification without alignment. J Real-Time Image Proc 12, 8 (2020). https:// doi.org/10.1007/s11554-020-01037-Z

2. Su, Y.S., Suen, H.Y., Hung, K.E.: Predicting behavioral competencies automatically from facial expressions in real-time videorecorded interviews. J Real-Time Image Proc (2021). https://doi. org/10.1007/s11554-021-01071-5

3. Chang, J., Dong, N., Li, D., et al.: Metric learning with generator for closed loop detection in VSLAM. J Real-Time Image Proc (2021). https://doi.org/10.1007/s11554-020-01067-7

4. Neff, C., Sheth, A., Furgurson, S., Middleton, J., Tabkhi, H.: EfficientHRNet: efficient and scalable high-resolution networks for real-time multi-person $2 \mathrm{D}$ human pose estimation. J Real-Time Image Proc (2021).

5. Singh, P., Diwakar, M., Cheng, X., et al.: A new wavelet-based multi-focus image fusion technique using method noise and anisotropic diffusion for real-time surveillance application. J Real-Time Image Proc (2021). https://doi.org/10.1007/s11554-021-01125-8

6. Gan, B., Zhang, C., Chen, Y., et al.: Research on role modeling and behavior control of virtual reality animation interactive system in internet of things. J Real-Time Image Proc (2020). https:// doi.org/10.1007/s11554-020-01046-y

7. Bacanin, N., Bezdan, T., Venkatachalam, K., et al.: Optimized convolutional neural network by firefly algorithm for magnetic resonance image classification of glioma brain tumor grade. J Real-Time Image Proc (2021). https://doi.org/10.1007/ s11554-021-01106-x

8. Chagas, J.V.S.D., de Rodrigues, A.D., Ivo, R.F., et al.: A new approach for the detection of pneumonia in children using CXR images based on an real-time IoT system. J Real-Time Image Proc (2021). https://doi.org/10.1007/s11554-021-01086-y

9. Tong, Y., Sun, W.: The role of film and television big data in realtime image detection and processing in the Internet of Things era. J Real-Time Image Proc (2021). https://doi.org/10.1007/ s11554-021-01105-y

10. Ahmad, M., Ahmed, I., Jeon, G.: An IoT-enabled real-time overhead view person detection system based on Cascade-RCNN and transfer learning. J Real-Time Image Proc (2021). https://doi.org/ 10.1007/s11554-021-01103-0

11. Jhong, S.Y., Chen, Y.Y., Hsia, C.H., Lin, S.C., Hsu, K.H., Lai, C.F.: Nighttime object detection system with lightweight deep network for internet of vehicles. J Real-Time Image Proc (2021).

Publisher's Note Springer Nature remains neutral with regard to jurisdictional claims in published maps and institutional affiliations. 